ALEA, Lat. Am. J. Probab. Math. Stat. 14, 177-199 (2017)

DOI: 10.30757/ALEA.v14-11

\title{
Exponential convergence to quasi-stationary distribution for absorbed one-dimensional diffusions with killing
}

\section{Nicolas Champagnat and Denis Villemonais}

IECL, Université de Lorraine, Site de Nancy, B.P. 70239, F-54506

Vandœuvre-lès-Nancy Cedex, France

CNRS, IECL, UMR 7502, Vandœuvre-lès-Nancy, F-54506, France

Inria, TOSCA team, Villers-lès-Nancy, F-54600, France.

E-mail address: Nicolas.Champagnat@inria.fr

IECL, Université de Lorraine, Site de Nancy, B.P. 70239, F-54506

Vandœuvre-lès-Nancy Cedex, France

CNRS, IECL, UMR 7502, Vandœuvre-lès-Nancy, F-54506, France

Inria, TOSCA team, Villers-lès-Nancy, F-54600, France.

E-mail address: Denis.Villemonais@univ-lorraine.fr

\begin{abstract}
This article studies the quasi-stationary behavior of absorbed onedimensional diffusion processes with killing on $[0, \infty)$. We obtain criteria for the exponential convergence to a unique quasi-stationary distribution in total variation, uniformly with respect to the initial distribution. Our approach is based on probabilistic and coupling methods, contrary to the classical approach based on spectral theory results. Our general criteria apply in the case where $\infty$ is entrance and 0 either regular or exit, and are proved to be satisfied under several explicit assumptions expressed only in terms of the speed and killing measures. We also obtain exponential ergodicity results on the $Q$-process. We provide several examples and extensions, including diffusions with singular speed and killing measures, general models of population dynamics, drifted Brownian motions and some onedimensional processes with jumps.
\end{abstract}

Received by the editors October 20, 2015; accepted December 3, 2016.

2010 Mathematics Subject Classification. Primary: 60J60, 60J70, 37A25, 60B10, 60F99. Secondary: 60G44, 60J75.

Key words and phrases. diffusions, one-dimensional diffusions with killing, absorbed process, quasi-stationary distribution, $Q$-process; uniform exponential mixing property, one dimensional processes with jumps. 


\section{Introduction}

This article studies the quasi-stationary behavior of general one-dimensional diffusion processes with killing in an interval $E$ of $\mathbb{R}$, absorbed at its finite boundaries. When the process is killed or absorbed, it is sent to some cemetary point $\partial$. This covers the case of solutions to one-dimensional stochastic differential equations (SDE) with space-dependent killing rate, but also of diffusions with singular speed and killing measures.

We recall that a quasi-stationary distribution for a continuous-time Markov process $\left(X_{t}, t \geq 0\right)$ on the state space $E \cup\{\partial\}$, is a probability measure $\alpha$ on $E$ such that

$$
\mathbb{P}_{\alpha}\left(X_{t} \in \cdot \mid t<\tau_{\partial}\right)=\alpha(\cdot), \quad \forall t \geq 0,
$$

where $\mathbb{P}_{\alpha}$ denotes the distribution of the process $X$ given that $X_{0}$ has distribution $\alpha$, and

$$
\tau_{\partial}:=\inf \left\{t \geq 0: X_{t}=\partial\right\} .
$$

We refer to Méléard and Villemonais (2012); van Doorn and Pollett (2013); Pollett (2015) for general introductions to the topic.

Our goal is to give conditions ensuring the existence of a unique quasi-limiting distribution $\alpha$ on $E$, i.e. a probability measure $\alpha$ such that for all probability measures $\mu$ on $E$ such that $\mu(\stackrel{\circ}{E})>0$ and all $A \subset E$ measurable,

$$
\lim _{t \rightarrow+\infty} \mathbb{P}_{\mu}\left(X_{t} \in A \mid t<\tau_{\partial}\right)=\alpha(A),
$$

where, in addition, the convergence is exponential and uniform with respect to $\mu$ and $A$. In particular, $\alpha$ is the unique quasi-stationary distribution.

This topic has been extensively studied for one-dimensional diffusions without killing in Cattiaux et al. (2009); Littin (2012); Miura (2014); Champagnat and Villemonais (2016b) Collet et al. (2013), where nearly optimal criteria are obtained. The case with killing is more complex and the existing results cover less general situations Steinsaltz and Evans (2007); Kolb and Steinsaltz (2012). In particular, these references are restricted to the study of solutions to SDEs with continuous absorption rate up to the boundary of $E$, and let the questions of uniqueness of the quasi-stationary distribution and of convergence in (1.1) open.

The present paper is focused on the case of a diffusion on $E=[0,+\infty)$ absorbed at 0 with scale function $s$, speed measure $m$ and killing measure $k$, assuming that killing corresponds to an immediate jump to $\partial=0$. We consider the situation where $\infty$ is an entrance boundary and 0 is either exit or regular. Our results easily extend to cases of bounded intervals with reachable boundaries.

We give two criteria, each of them involving one condition concerning the diffusion (without killing) with scale function $s$ and speed measure $m$, and another condition on the killing time. The condition on the diffusion without killing comes from Champagnat and Villemonais (2016b) and covers nearly all one-dimensional diffusions on $[0, \infty)$ such that $\infty$ is an entrance boundary and a.s. absorbed in finite time at 0 . The conditions on the killing time only concern the behavior of the diffusion and of the killing measure in the neighborhood of 0 , as soon as $\infty$ is an entrance boundary. In order to apply these results to practical situations, we provide several explicit criteria ensuring all these conditions and a series of examples that enter our setting. 
This contribution improves known results in several directions. First, it covers situations of diffusion processes which are not solutions to SDEs and cases of irregular and unbounded killing rates. Secondly, aside from proving the existence of a quasi-stationary distribution, we also obtain exponential convergence of conditional distributions. In particular, all the initial distributions of $X$ belong to the domain of attraction of the quasi-stationary distribution. This is of great importance in applications, since in practice, one usually has no precise estimate of the initial distribution. In addition, our estimates of convergence are exponential and uniform in total variation norm, and hence provide a uniform bound for the time needed to observe stabilisation of the conditional distribution of the process, regardless of the initial distribution (see also the discussion in Méléard and Villemonais, 2012, Ex. 2).

The main methodological novelty of our proofs relies on its purely probabilistic approach. We do not use any spectral theoretical result (typically, Sturm-Liouville theory), which are the key tool of all the previously cited works on diffusions, except Champagnat and Villemonais (2016b). Instead, we use criteria for general Markov processes proved in Champagnat and Villemonais (2016a), based on coupling and Dobrushin coefficient techniques. These criteria also imply exponential ergodicity for the $Q$-process, defined as the process $X$ conditioned to never be absorbed. The generality and flexibility of this approach also allow to cover, without substantial modification of the arguments, many situations where the spectral theory has received much less attention, such as one-dimensional diffusions with jumps and killing.

For our study, we need to give a probabilistic formulation of the property that $\infty$ is entrance. In the case without killing, this is known as the classical property of coming down from infinity (see e.g. Cattiaux et al., 2009; Champagnat and Villemonais, 2016b). For a diffusion with killing, we show that a process with entrance boundary at $\infty$ comes down from infinity before killing in the sense that the diffusion started from $+\infty$ hits 0 in finite time before killing with positive probability.

The paper is organized as follows. In Section 2, we precisely define the absorbed diffusion processes with killing under study and give their construction as timechanged Brownian motions. Although quite natural, this alternative construction of killed diffusions is not given in classical references. Section 3.1 contains the statement of our main results on the exponential convergence of conditional distributions, the asymptotic behavior of the probability of survival and the existence and ergodicity of the $Q$-process. In Subsection 3.2, we give explicit criteria ensuring the conditions of Section 3.1. We focus in Sections 2 and 3 on diffusions on natural scale. The extension to general diffusions is given in Section 4.1, together with a series of examples of diffusions which are not solutions to SDEs, of diffusions with unbounded or irregular killing rates and of one-dimensional processes with killing and jumps. Section 4.2 is devoted to the study of models of population dynamics of the form

$$
d Y_{t}=\sqrt{Y_{t}} d B_{t}+Y_{t} h\left(Y_{t}\right) d t
$$

and Section 4.3 to drifted Brownian motions, which are the basic models of Steinsaltz and Evans (2007); Kolb and Steinsaltz (2012). Section 5 concerns the property of coming down from infinity before killing. Finally, Section 6 gives the proofs of our main results. 


\section{Absorbed diffusion processes with killing}

Our goal is to construct diffusion processes with killing on $[0,+\infty)$, absorbed at $\partial=0$. The typical situation corresponds to stochastic population dynamics of continuous densities with possible continuous or sudden extinction.

We recall that a stochastic process $\left(X_{t}, t \geq 0\right)$ on $[0,+\infty)$ is called a diffusion (without killing) if it has a.s. continuous paths, satisfies the strong Markov property and is regular. By regular, we mean that for all $x \in(0, \infty)$ and $y \in[0, \infty)$, $\mathbb{P}_{x}\left(T_{y}<\infty\right)>0$, where $T_{y}$ is the first hitting time of $y$ by the process $X$. Given such a process, there exists a continuous and strictly increasing function $s$ on $[0, \infty)$, called the scale function, such that $\left(s\left(X_{t \wedge T_{0}}\right), t \geq 0\right)$ is a local martingale, see Freedman (1983). The stochastic process $\left(s\left(X_{t}\right), t \geq 0\right)$ is itself a diffusion process with identity scale function. Replacing $\left(X_{t}, t \geq 0\right)$ by $\left(s\left(X_{t}\right), t \geq 0\right)$, we can assume without loss of generality that $s(x)=x$.

To such a process $X$ on natural scale, one can associate a unique locally finite positive measure $m(d x)$ on $(0, \infty)$, called the speed measure of $X$, which gives positive mass to any open subset of $(0,+\infty)$ and such that $X_{t}=B_{\sigma_{t}}$ for all $t \geq 0$ for some standard Brownian motion $B$, where

$$
\sigma_{t}=\inf \left\{s>0: A_{s}>t\right\}, \quad \text { with } A_{s}=\int_{0}^{\infty} L_{s}^{x} m(d x)
$$

and $L^{x}$ is the local time of $B$ at level $x$. Conversely, given any positive locally finite measure $m$ on $(0, \infty)$ giving positive mass to any open subset of $(0, \infty)$, any such time change of a Brownian motion defines a regular diffusion on $[0, \infty)$, see Kallenberg (2002, Thm.23.9). Note that, since $\sigma_{t}$ is continuous and since $X_{t}=B_{\sigma_{t}}$ for all $t \geq 0$ a.s., we have

$$
\sigma_{T_{0}}=T_{0}^{B}, \quad \text { or, equivalently, } T_{0}=A_{T_{0}^{B}},
$$

where $T_{x}^{B}$ is the first hitting time of $x \in \mathbb{R}$ by the process $B$.

In this work, we study diffusion processes on $[0,+\infty)$ with killing as defined in Itô and McKean Jr. (1974). As above, we can assume without loss of generality that the diffusion is on natural scale. We show below that such a process can be obtained from an explicit pathwise construction from a given Brownian motion $\left(B_{t}, t \geq 0\right)$ and an independent exponential random variable $\mathcal{E}$ of parameter 1 . Although this construction is quite natural, this is not done in the classical references Itô and McKean Jr. (1974); Freedman (1983); Méléard (1986); Kallenberg (2002).

Let $k$ and $m$ be two positive locally finite measures on $(0,+\infty)$, such that $m$ gives positive mass to any open subset of $(0,+\infty)$. The measures $m$ and $k$ will be referred to as the speed measure and the killing measure, respectively, of the diffusion with killing.

We first define the diffusion process on natural scale without killing as above by

$$
\tilde{X}_{t}:=B_{\sigma_{t}}, \quad \forall t \geq 0,
$$

where $\sigma_{t}$ is defined by (2.1). Next, we define for all $t \geq 0$

$$
\kappa_{t}:=\int_{0}^{\infty} L_{\sigma_{t}}^{y} k(d y), \quad \forall t \geq 0 .
$$


It follows from the definition of the local time $\left(L_{t}^{\tilde{X}, x}, t \geq 0, x \geq 0\right)$ of the process $\widetilde{X}$ of Freedman (1983, p. 160) that

$$
\kappa_{t}=\int_{0}^{\infty} L_{t}^{\widetilde{X}, y} k(d y) .
$$

Let $\mathcal{E}$ be an exponential r.v. of parameter 1 independent of $B$. Then we define

$$
\tau_{\kappa}:=\inf \left\{t \geq 0: \kappa_{t} \geq \mathcal{E}\right\} \quad \text { and } \quad \widetilde{T}_{0}:=\inf \left\{t \geq 0: \widetilde{X}_{t}=0\right\} .
$$

Our goal is to construct a process $X$ that can hit 0 either continuously following the path of $\widetilde{X}$, or discontinuously when it is killed at time $\tau_{\kappa}$. This leads to the following definition of the time of discontinuous absorption (or killing time) $\tau_{\partial}^{d}$ and the time of continuous absorption $\tau_{\partial}^{c}$ :

$$
\tau_{\partial}^{d}:=\left\{\begin{array}{ll}
\tau_{\kappa} & \text { if } \tau_{\kappa}<\widetilde{T}_{0}, \\
+\infty & \text { if } \widetilde{T}_{0} \leq \tau_{\kappa}
\end{array} \quad \text { and } \quad \tau_{\partial}^{c}:= \begin{cases}+\infty & \text { if } \tau_{\kappa}<\widetilde{T}_{0}, \\
\widetilde{T}_{0} & \text { if } \widetilde{T}_{0} \leq \tau_{\kappa} .\end{cases}\right.
$$

The absorption time $\tau_{\partial}$ is then defined as

$$
\tau_{\partial}:=\tau_{\partial}^{d} \wedge \tau_{\partial}^{c}
$$

Then, the absorbed diffusion process with killing $X$ is defined as

$$
X_{t}:= \begin{cases}\widetilde{X}_{t} & \text { if } t<\tau_{\partial} \\ \partial=0 & \text { otherwise. }\end{cases}
$$

Proposition 2.1. The process $\left(X_{t}, t \geq 0\right)$ defined in (2.6) is a diffusion process with killing on natural scale, with speed measure $m$ and killing measure $k$, as defined in Itô and McKean Jr. (1974).

Proof: Note that

$$
\kappa_{t}=\int_{0}^{\infty} L_{t}^{X, y} k(d y), \quad \forall t<\tau_{\kappa},
$$

since $X$ and $\tilde{X}$ coincide before $\tau_{\kappa}$. Since $E$ is independent of $B$,

$$
\mathbb{P}\left(\tau_{\kappa}>t \mid \tilde{X}_{t}, t \geq 0\right)=\mathbb{P}\left(\tau_{\kappa}>t \mid B_{s}, s \geq 0\right)=\exp \left(-\int_{0}^{\infty} L_{t}^{X, y} k(d y)\right) .
$$

This is exactly the definition of the distribution of the killing time of the diffusion process with killing of Itô and McKean Jr. (1974, p. 179).

In particular, in the case where $k$ is absolutely continuous with respect to $m$, the killing rate of the diffusion at position $x$ (in the sense e.g. of Kolb and Steinsaltz, 2012 ) is given by $\frac{d k}{d m}(x)$. This can be deduced from (2.7) and from the occupation time formula of Freedman (1983).

In addition, the property of regularity of the diffusion with killing can be easily checked from this definition, as shown in the next proposition.

Proposition 2.2. For all $x, y>0$,

$$
\mathbb{P}_{x}\left(T_{y}<\infty\right)>0
$$

and for all $x>0$ and $t>0$,

$$
\mathbb{P}_{x}\left(\tau_{\partial}>t\right)>0, \quad \forall x>0, \forall t \geq 0 .
$$


Proof: Fix $x \neq y$. On the event $\left\{\widetilde{T}_{y}<\infty\right\}$, we have $\sigma_{\widetilde{T}_{y}}=T_{y}^{B}<T_{0}^{B}$. In particular, the infimum of $B_{s}$ over $\left[0, T_{y}^{B}\right]$ is positive, and its maximum is finite. Hence the function $y \mapsto L_{T_{y}^{B}}^{y}$ is a.s. continuous with compact support in $(0,+\infty)$, hence a.s. bounded, on the event $\left\{\widetilde{T}_{y}<\infty\right\}$. Since $k$ is locally finite on $(0,+\infty)$ and $\mathbb{P}_{x}\left(\widetilde{T}_{y}<\infty\right)>0$, this implies that

$$
\begin{aligned}
\mathbb{P}_{x}\left(T_{y}<\infty\right) & =\mathbb{P}_{x}\left(\widetilde{T}_{y}<\infty \text { and } \kappa_{\widetilde{T}_{y}}<\mathcal{E}\right) \\
& =\mathbb{E}_{x}\left[\mathbb{1}_{\widetilde{T}_{y}<\infty} \exp \left(-\int_{0}^{\infty} L_{T_{y}^{B}}^{y} k(d y)\right)\right]>0 .
\end{aligned}
$$

To prove (2.8), we fix $t>0$. As above, a.s. on the event $\left\{t<\tau_{\partial}^{c}\right\}=\left\{\sigma_{t}<T_{0}^{B}\right\}$, the function $y \mapsto L_{\sigma_{t}}^{y}$ is continuous with compact support on $(0,+\infty)$. Since $k$ is locally finite on $(0,+\infty)$, we deduce that $\kappa_{t}<\infty$ a.s. Since in addition $\mathbb{P}_{x}\left(\widetilde{X}_{t}>0\right)>0$ for all $t>0,(2.8)$ follows.

In the sequel, following the terminology of Feller (1952), we assume that $+\infty$ is an entrance boundary, or equivalently (for diffusion processes on natural scale)

$$
\int_{1}^{\infty} y(d m(y)+d k(y))<\infty
$$

and that 0 is either regular or an exit point, or equivalently

$$
\int_{0}^{1} y(d m(y)+d k(y))<\infty .
$$

Note that, in the case where $k=0, X=\widetilde{X}$ is a diffusion without killing. In this case, the assumption $\int_{1}^{\infty} y d m(y)<\infty$ corresponds to the fact that $X$ comes down from infinity Champagnat and Villemonais (2016b), i.e. that there exist $t>0$ and $y>0$ such that

$$
\inf _{x>y} \mathbb{P}_{x}\left(T_{y}<t\right)>0
$$

and $\int_{0}^{1} y d m(y)<\infty$ is equivalent to assuming that $\tau_{\partial}=\tau_{\partial}^{c}<\infty$ a.s.

Further general properties of diffusion processes with killing are studied in Section 5 , related to the notion of coming down from infinity.

\section{Quasi-stationary distributions for diffusion processes with killing}

We consider as above a diffusion process $X$ on $[0,+\infty)$ with killing, on natural scale, with speed measure $m(d x)$ and killing measure $k(d x)$.

3.1. Exponential convergence to quasi-stationary distribution. We provide here sufficient criteria ensuring the existence of a unique quasi-stationary distribution for $X$, with exponential convergence of conditional distributions in total variation. Both criteria involve the diffusion process without killing $\widetilde{X}$ of Section 2.

Condition (C) Assume that $\int_{0}^{\infty} y(m(d y)+k(d y))<\infty$ and that there exist two constants $t_{1}, A>0$ such that

$$
\mathbb{P}_{x}\left(t_{1}<\widetilde{T}_{0}\right) \leq A x, \quad \forall x>0
$$


and

$$
\mathbb{P}_{x}\left(\tau_{\partial}^{d}<\tau_{\partial}^{c}\right) \leq A x, \quad \forall x>0
$$

Condition (C') Assume that $\int_{0}^{\infty} y(m(d y)+k(d y))<\infty$ and that there exist three constants $t_{1}, A, \varepsilon>0$ such that

$$
\mathbb{P}_{x}\left(t_{1}<\widetilde{T}_{0}\right) \leq A x, \quad \forall x>0
$$

and $k$ is absolutely continuous w.r.t. $m$ on $(0, \varepsilon)$ and satisfies

$$
\frac{d k}{d m}(x) \leq A, \quad \forall x \in(0, \varepsilon) .
$$

We give practical criteria to check Conditions (C) and (C') is Subsection 3.2 and we give examples of applications and compare with existing results in Section 4.

Theorem 3.1. Assume that $X$ is a one-dimensional diffusion on natural scale with speed measure $m$ and killing measure $k$. If Assumption (C) or Assumption (C') is satisfied, then there exists a unique probability measure $\alpha$ on $(0,+\infty)$ and two constants $C, \gamma>0$ such that, for all initial distribution $\mu$ on $E$ such that $\mu(\{0\})<1$,

$$
\left\|\mathbb{P}_{\mu}\left(X_{t} \in \cdot \mid t<\tau_{\partial}\right)-\alpha(\cdot)\right\|_{T V} \leq C e^{-\gamma t}, \forall t \geq 0 .
$$

In this case, $\alpha$ is the unique quasi-stationary distribution for the process.

We recall that the quasi-stationary distribution $\alpha$ of Thm. 3.1 satisfies the following classical property: there exists $\lambda_{0}>0$ such that $\mathbb{P}_{\alpha}\left(t<\tau_{\partial}\right)=e^{-\lambda_{0} t}$. The same assumptions also entail the following result on the asymptotic behavior of absorption probabilities.

Proposition 3.2. Assume that $X$ is a one-dimensional diffusion on natural scale with speed measure $m$ and killing measure $k$. If Assumption (C) or Assumption (C') is satisfied, then there exists a bounded function $\eta:(0, \infty) \rightarrow(0, \infty)$ such that

$$
\eta(x)=\lim _{t \rightarrow \infty} \frac{\mathbb{P}_{x}\left(t<\tau_{\partial}\right)}{\mathbb{P}_{\alpha}\left(t<\tau_{\partial}\right)}=\lim _{t \rightarrow+\infty} e^{\lambda_{0} t} \mathbb{P}_{x}\left(t<\tau_{\partial}\right),
$$

where the convergence holds for the uniform norm. Moreover, $\alpha(\eta)=1, \eta(x) \leq$ $C x$ for all $x \geq 0$ and some constant $C>0$, and $\eta$ belongs to the domain of the infinitesimal generator $L$ of $X$ on the set of bounded measurable functions on $[0,+\infty)$ equipped with uniform norm and

$$
L \eta=-\lambda_{0} \eta \text {. }
$$

Still under the same assumptions, we also obtain the exponential ergodicity of the $Q$-process, which is defined as the process $X$ conditioned to never be absorbed.

Theorem 3.3. Assume that $X$ is a one-dimensional diffusion on natural scale with speed measure $m$ and killing measure $k$. If Assumption $(\mathrm{C})$ or Assumption ( $\left.\mathrm{C}^{\prime}\right)$ is satisfied, then the three following properties hold true.

(i) Existence of the $Q$-process.: There exists a family $\left(\mathbb{Q}_{x}\right)_{x>0}$ of probability measures on $\Omega$ defined by

$$
\lim _{t \rightarrow+\infty} \mathbb{P}_{x}\left(A \mid t<\tau_{\partial}\right)=\mathbb{Q}_{x}(A)
$$

for all $\mathcal{F}_{s}$-measurable set $A$, for all $s \geq 0$. The process $\left(\Omega,\left(\mathcal{F}_{t}\right)_{t \geq 0},\left(X_{t}\right)_{t \geq 0}\right.$, $\left.\left(\mathbb{Q}_{x}\right)_{x>0}\right)$ is a $(0, \infty)$-valued homogeneous strong Markov process. 
(ii) Transition kernel.: $\quad$ The transition kernel of the Markov process $X$ under $\left(\mathbb{Q}_{x}\right)_{x>0}$ is given by

$$
\tilde{p}(x ; t, d y)=e^{\lambda_{0} t} \frac{\eta(y)}{\eta(x)} p(x ; t, d y) \mathbb{1}_{y>0}, \quad \forall t \geq 0, x>0,
$$

where $p(x ; t, d y)$ is the transition distribution of the diffusion with killing $X$.

(iii) Exponential ergodicity.: The probability measure $\beta$ on $(0,+\infty)$ defined by

$$
\beta(d x)=\eta(x) \alpha(d x) .
$$

is the unique invariant distribution of $X$ under $\mathbb{Q}$. Moreover, there exist positive constants $C, \gamma$ such that, for any initial distributions $\mu_{1}, \mu_{2}$ on $(0,+\infty)$,

$$
\left\|\mathbb{Q}_{\mu_{1}}\left(X_{t} \in \cdot\right)-\mathbb{Q}_{\mu_{2}}\left(X_{t} \in \cdot\right)\right\|_{T V} \leq C e^{-\gamma t}\left\|\mu_{1}-\mu_{2}\right\|_{T V},
$$

where $\mathbb{Q}_{\mu}=\int_{(0, \infty)} \mathbb{Q}_{x} \mu(d x)$.

The proofs of these results are given in Section 6 .

3.2. On Conditions $(C)$ and $\left(C^{\prime}\right)$. The first step to check Conditions $(\mathrm{C})$ or $\left(\mathrm{C}^{\prime}\right)$ is to check (3.1). This condition is the one ensuring exponential convergence of conditional distributions of general diffusion processes without killing (see Champagnat and Villemonais, 2016b, Thm.4.1), and it has been extensively studied in Champagnat and Villemonais (2016b). We recall here the most general criterion, which applies to all practical cases where $\infty$ is entrance and 0 is exit or natural, since it fails only when $m$ has strong oscillations near 0 (see Champagnat and Villemonais, 2016b, Rk. 6).

Theorem 3.4 (Champagnat and Villemonais, 2016b, Thm 4.6). Assume that $\int_{0}^{\infty} y m(d y)<\infty$ and

$$
\int_{0}^{1} \frac{1}{x} \sup _{y \leq x}\left(\frac{1}{y} \int_{(0, y)} z^{2} m(d z)\right) d x<\infty .
$$

Then, for all $t>0$, there exists $A_{t}<\infty$ such that

$$
\mathbb{P}_{x}\left(t<\widetilde{T}_{0}\right) \leq A_{t} x, \forall x>0 .
$$

Using this result, the remaining conditions in $(\mathrm{C})$ and $\left(\mathrm{C}^{\prime}\right)$ only deal with the behavior of the diffusion process $X$ near 0 . We provide two sufficient criteria for (3.2) in Condition (C) in Proposition 3.5 and Proposition 3.6. Note that checking property (3.3) in Condition (C') from given measures $m$ and $k$ is straightforward.

Proposition 3.5. Assume that $\int_{0}^{\infty} y k(d y)<\infty$ and that there exist $\varepsilon>0$ and a constant $C>0$ such that $k$ is absolutely continuous w.r.t. the Lebesgue measure $\Lambda$ on $(0, \varepsilon)$ and such that

$$
\frac{d k}{d \Lambda}(x) \leq \frac{C}{x}, \forall x \in(0, \varepsilon) .
$$

Then there exists a positive constant $A>0$ such that

$$
\mathbb{P}_{x}\left(\tau_{\partial}^{d}<\tau_{\partial}^{c}\right) \leq A x
$$


Note that, contrary to Condition (C'), the previous criterion does not require the killing rate $d k / d m$ of the diffusion process to be bounded on a neighborhood of 0 . It is actually independent of the measure $m$. For instance, if $m(d x)=d x$ and $k(d x)=\frac{1}{x} d x$ on a neighborhood of 0 , then the assumptions of Proposition 3.5 are satisfied while $d k / d m(x)=1 / x$ is unbounded near 0 .

Proof of Proposition 3.5: We have from (2.3)

$$
\mathbb{P}_{x}\left(\tau_{\partial}^{d}<\tau_{\partial}^{c}\right)=\mathbb{P}_{x}\left(\kappa_{\widetilde{T}_{0}}>\mathcal{E}\right)=\mathbb{P}_{x}\left(\int_{0}^{\infty} L_{\widetilde{T}_{0}}^{y} d k(y)>\mathcal{E}\right)
$$

Hence, by (2.2),

$$
\mathbb{P}_{x}\left(\tau_{\partial}^{d}<\tau_{\partial}^{c}\right)=\mathbb{P}_{x}\left(\int_{0}^{\infty} L_{T_{0}^{B}}^{y} d k(y)>\mathcal{E}\right)=\mathbb{P}_{x}^{Y}\left(T_{0}^{Y}>\mathcal{E}\right),
$$

where $Y$ is a diffusion process on $(0, \infty)$ on natural scale with speed measure $k$, absorbed at 0 and without killing. We have

$$
\begin{aligned}
\mathbb{P}_{x}^{Y}\left(T_{0}^{Y}>\mathcal{E}\right) & \leq \mathbb{P}_{x}^{Y}\left(\mathcal{E}<T_{0}^{Y}<T_{\varepsilon}^{Y}\right)+\mathbb{P}_{x}^{Y}\left(T_{\varepsilon}^{Y} \leq T_{0}^{Y}\right) \\
& \leq \mathbb{P}_{x}^{Y}\left(\mathcal{E}<T_{0}^{Y}<T_{\varepsilon}^{Y}\right)+x / \varepsilon
\end{aligned}
$$

since $Y$ is on natural scale and hence is a local martingale. As a consequence, it only remains to prove that $\mathbb{P}_{x}^{Y}\left(\mathcal{E}<T_{0}^{Y}<T_{\varepsilon}^{Y}\right) \leq A x$. Because of our assumption (3.8),

$$
\begin{aligned}
\mathbb{P}_{x}^{Y}\left(\mathcal{E}<T_{0}^{Y}<T_{\varepsilon}^{Y}\right) & =\mathbb{P}_{x}\left(\left\{T_{0}^{B}<T_{\varepsilon}^{B}\right\} \cap\left\{\int_{0}^{\infty} L_{T_{0}^{B}}^{y} d k(y)>\mathcal{E}\right\}\right) \\
& \leq \mathbb{P}_{x}\left(\left\{T_{0}^{B}<T_{\varepsilon}^{B}\right\} \cap\left\{\int_{0}^{\infty} L_{T_{0}^{B}}^{y} \frac{d y}{y}>\mathcal{E} / C\right\}\right) \\
& \leq \mathbb{P}_{x}\left(\int_{0}^{\infty} L_{T_{0}^{B}}^{y} \frac{d y}{y}>\mathcal{E} / C\right)=1-v(x)
\end{aligned}
$$

where $v(x)=\mathbb{P}_{x}^{Z}\left(T_{0}^{Z} \leq \mathcal{E} / C\right)$ with $Z$ the diffusion on $(0,+\infty)$ on natural scale and with speed measure $(1 / y) d y$, i.e. solution to the SDE

$$
d Z_{t}=\sqrt{Z_{t}} d W_{t}
$$

for $\left(W_{t}\right)_{t \geq 0}$ a standard Brownian motion. The diffusion process $\left(Z_{t}\right)_{t \geq 0}$ is a continuous state branching process (Feller diffusion), hence by the branching property,

$$
v(x+y)=v(x) v(y), \forall x, y \geq 0 .
$$

Since $v(x)$ is non-increasing and $v(0)=1$, we deduce from standard arguments that there exists a constant $A>0$ such that

$$
v(x)=e^{-A x} .
$$

Proposition 3.5 follows.

Proposition 3.6. Assume that $\int_{1}^{\infty} y k(d y)<\infty$ and $k([0,1])<\infty$. Then there exists a positive constant $A>0$ such that

$$
\mathbb{P}_{x}\left(\tau_{\partial}^{d}<\tau_{\partial}^{c}\right) \leq A x
$$

We emphasize that measures $k$ which are not absolutely continuous w.r.t. the Lebesgue measure are of course also covered by Prop. 3.6. Note also that Prop. 3.6 does not imply Prop. 3.5 since the killing measure $d k(x)=(1 / x) d \Lambda(x) \mathbb{1}_{x \in(0,1)}$ is not finite. 
Proof of Proposition 3.6: As in the beginning of the proof of Proposition 3.5, we obtain

$$
\begin{aligned}
\mathbb{P}_{x}\left(\tau_{\partial}^{c}<\tau_{\partial}^{d}\right) & =\mathbb{P}_{x}\left(\int_{0}^{\infty} L_{T_{0}^{B}}^{y} d k(y)<\mathcal{E}\right) \\
& =\mathbb{E}_{x}\left[\exp \left(-\int_{0}^{\infty} L_{T_{0}^{B}}^{y} d k(y)\right)\right] \\
& \geq 1-\mathbb{E}_{x}\left(\int_{0}^{\infty} L_{T_{0}^{B}}^{y} d k(y)\right) \\
& =1-\mathbb{E}_{x}^{Y}\left(T_{0} \mid Y_{0}=x\right)
\end{aligned}
$$

where $\mathbb{E}^{Y}$ denotes the expectation w.r.t. the law of a diffusion process $Y$ on $(0,+\infty)$ on natural scale, without killing and whose speed measure is $k(d y)$. Finally, using classical Green formula for diffusion processes without killing applied to $Y$, we conclude that

$$
\mathbb{P}_{x}\left(\tau_{\partial}^{c}<\tau_{\partial}^{d}\right) \geq 1-\int_{0}^{\infty}(x \wedge y) k(d y) \geq 1-x k([0,1])-x \int_{1}^{\infty} y k(d y) \geq 1-C x .
$$

\section{Examples and comparison with existing results}

4.1. On general diffusions. Let us first recall that our results also cover the case of general (i.e. not necessarily on natural scale) killed diffusion processes $\left(Y_{t}, t \geq 0\right)$ on $[0, \infty)$ with speed measure $m_{Y}$ and killing measure $k_{Y}$, such that the diffusion without killing $\widetilde{Y}$ hits 0 in a.s. finite time. Under these assumptions, there exists a continuous and strictly increasing scale function $s:[0, \infty) \rightarrow[0, \infty)$ of the process $Y$ such that $s(0)=0$ and $s(\infty)=\infty$, and our results apply to the process $X_{t}=s\left(Y_{t}\right)$ on natural scale, whose speed measure and killing measure are respectively given by

$$
m_{X}(d x)=m_{Y} * s(d x) \quad \text { and } \quad k_{X}(d x)=k_{Y} * s(d x),
$$

where $m_{Y} * s$ and $k_{Y} * s$ denote the pushforward measures of $m_{Y}$ and $k_{Y}$ through the function $s$ (the formula for $m_{X}$ can be found in Revuz and Yor (1999, Thm. VII.3.6) and for the measure $k_{X}$, one may use for example (2.3) and the fact that $L_{t}^{y, \widetilde{Y}}=$ $L_{t}^{s(y), \widetilde{X}}$ which follows from the occupation time formula of Freedman (1983, p. 160)).

Hence $X$ satisfies $(\mathrm{C})$ or $\left(\mathrm{C}^{\prime}\right)$ if and only if $Y$ satisfies the following Conditions (D) or (D') respectively. In particular, the conclusions of Theorem 3.1 apply to $Y$ if (D) or (D') is fulfilled.

Condition (D) Assume that $\int_{0}^{\infty} s(y)\left(m_{Y}(d y)+k_{Y}(d y)\right)<\infty$. Assume also that there exist two constants $t_{1}, A>0$ such that

$$
\mathbb{P}\left(t_{1}<\widetilde{T}_{0} \mid Y_{0}=y\right) \leq A s(y), \quad \forall y>0
$$

and

$$
\mathbb{P}\left(\tau_{\partial}^{d}<\tau_{\partial}^{c} \mid Y_{0}=y\right) \leq A s(y), \quad \forall y>0
$$

where the absorption times $\widetilde{T}_{0}, \tau_{\partial}^{d}$ and $\tau_{\partial}^{c}$ are constructed here from the processes $Y$ and $\tilde{Y}$. 
Condition (D') Assume that $\int_{0}^{\infty} s(y)\left(m_{Y}(d y)+k_{Y}(d y)\right)<\infty$. Assume also that there exist three constants $t_{1}, A, \varepsilon>0$ such that

$$
\mathbb{P}\left(t_{1}<\widetilde{T}_{0} \mid Y_{0}=y\right) \leq A s(y), \quad \forall y>0
$$

and $k_{Y}$ is absolutely continuous w.r.t. $m_{Y}$ on $(0, \varepsilon)$ and satisfies

$$
\frac{d k_{Y}}{d m_{Y}}(y) \leq A, \quad \forall y \in(0, \varepsilon)
$$

In particular, we deduce from (4.1) the following criteria for (D) and (D').

Proposition 4.1. Assume that $\int_{0}^{\infty} s(y)\left(m_{Y}(d y)+k_{Y}(d y)\right)<\infty$ and that there exist two constants $t_{1}, A>0$ such that

$$
\mathbb{P}\left(t_{1}<\widetilde{T}_{0} \mid Y_{0}=y\right) \leq A s(y), \quad \forall y>0 .
$$

Then

(i): If $k_{Y}([0,1])<\infty$, then $Y$ satisfies $(D)$.

(ii): If there exists $\varepsilon, \rho, C>0$ such that

$$
\frac{d k_{Y}}{d \Lambda}(y) \leq \frac{C}{s(y)}, \forall y \in(0, \varepsilon)
$$

and

$$
s(y)-s(x) \geq \rho(y-x), \quad \forall 0<x<y<\varepsilon,
$$

then $Y$ satisfies $(D)$.

(iii): If the killing rate $\kappa_{Y}(y):=\frac{d k_{Y}}{d m_{Y}}(y)$ exists and is uniformly bounded on $(0, \varepsilon)$ for some $\varepsilon>0$, then $Y$ satisfies ( $D$ ').

Proof: Point (i) follows immediately from Prop. 3.6 since $k_{X}([0, s(1)])=k_{Y}([0,1])$ $<\infty$.

For Point (ii), we deduce from (4.1) that

$$
\frac{d k_{X}}{d(\Lambda * s)} \leq \frac{C}{x}, \forall x \in(0, s(\varepsilon)) .
$$

In view of of Prop. 3.5, it only remains to prove that $d(\Lambda * s) / d \Lambda \leq C^{\prime}$ in the neighborhood of 0 . This is exactly (4.2).

Finaly, under the conditions of Point (iii), by (4.1), $\kappa_{X}(y):=\frac{d k_{X}}{d m_{X}}(x)$ exists and is uniformly bounded on $(0, s(\varepsilon))$. Hence $X$ satisfies $\left(\mathrm{C}^{\prime}\right)$.

Let us also mention that, as will appear clearly in the proofs of Section 6 , our methods can be easily extended to diffusion processes on a bounded interval, where one of the boundary point is an entrance boundary and the other is exit or regular, and also to cases where both boundary points are either exit or regular.

We provide now a few examples of diffusion processes that enter our setting (directly or considering the above extension).

Example 1. Let $X$ be a sticky Brownian motion on the interval $(-1,1)$ which is sticky at 0 , absorbed at -1 and 1 , and with measurable killing rate $\kappa:(-1,1) \mapsto$ $(0,+\infty)$ such that $\int_{(-1,1)} \kappa(x) d x<\infty$ and $\kappa(0)<\infty$. In this case $X$ is the diffusion with killing on natural scale with speed measure and killing measure

$$
m(d x)=\Lambda(d x)+\delta_{0}(d x) \quad \text { and } \quad k(d x)=\kappa(x) \Lambda(d x)+\kappa(0) \delta_{0}(d x) .
$$


Extending our result to diffusion processes on $(-1,1)$ with regular boundaries, we immediately deduce from Proposition 3.6 that $X$ admits a unique quasi-stationary distribution and satisfies (3.4).

Example 2. Let $Y$ be a solution to the SDE

$$
d Y_{t}=\sqrt{Y_{t}} d B_{t}+Y_{t}\left(r-c Y_{t}\right) d t
$$

where $r \in \mathbb{R}$ represents the individual growth rate without competition and $c>0$ governs the competitive density dependence in the growth rate. We assume that the diffusion process $Y$ is subject to the killing rate $\kappa(y)=\sin (1 / y) \vee \sqrt{y}$. Then $Y$ admits a unique quasi-stationary distribution and satisfies (3.4) (see Proposition 4.2 for a detailed study of this case in a more general setting).

In this example, 0 is an exit boundary and $\kappa$ is not continuous at 0 (see the comparison with the literature in Section 4.3).

Example 3. Let $Y$ be a solution to the SDE

$$
d Y_{t}=d B_{t}-Y_{t}^{2} d t
$$

absorbed at 0 . We may for example assume that the diffusion process $Y$ is subject to the non-negative killing rate $\kappa(y)=\frac{1}{\sqrt{y}} \vee \sqrt{y}$, or to the killing measure

$$
k(d y)=\sum_{n \in \mathbb{N}} b_{n} \delta_{a_{n}}(d y),
$$

for any bounded sequence $\left(a_{n}\right)_{n \in \mathbb{N}}$ and summable family $\left(b_{n}\right)_{n \in \mathbb{N}}$. In both cases, condition (D) is satisfied.

These two examples illustrate that our results also cover cases of unbounded (and even singular) killing rate $\kappa$.

Example 4. In addition, our method is general enough to apply to more complex processes. An example of diffusion without killing but with jumps is studied in Champagnat and Villemonais (2016b, Sec. 3.5.4). The extension to the case of diffusions with killing is straightforward using the same method: we consider a diffusion process on natural scale $\left(X_{t}, t \geq 0\right)$ on $[0, \infty)$ with speed measure $m$ and killing measure $k$ such that either Condition $(\mathrm{C})$ or $\left(\mathrm{C}^{\prime}\right)$ is satisfied. Let us denote by $\mathcal{L}$ the infinitesimal generator of $X$.

Our first example is the Markov process $\left(\widehat{X}_{t}, t \geq 0\right)$ with infinitesimal generator

$$
\widehat{\mathcal{L}} f(x)=\mathcal{L} f(x)+(f(x+1)-f(x)) \mathbb{1}_{x \geq 1},
$$

for all $f$ in the domain of $\mathcal{L}$. In other words, we consider a càdlàg process following a diffusion process with speed measure $m$ and killing measure $k$ between jump times, which occur at the jump times of an independent Poisson process $\left(N_{t}, t \geq 0\right)$ of rate 1 , with jump size +1 if the process is above 1 , and 0 otherwise. Then, a straightforward adaptation of Prop. 3.10 of Champagnat and Villemonais (2016b) implies that the conclusion of Thm. 3.1 holds for $\widehat{X}$.

Another simple example is given by the Markov process $\left(\widehat{X}_{t}, t \geq 0\right)$ with infinitesimal generator

$$
\widehat{\mathcal{L}} f(x)=\mathcal{L} f(x)+f(x+1)-f(x),
$$

for all $f$ in the domain of $\mathcal{L}$. Here, +1 jumps occur at Poisson times, regardless of the position of the process. Then, in the case where $(m, k)$ satisfies Condition $(\mathrm{C})$ 
and if $\frac{d m}{d \Lambda}(x) \leq \frac{C}{x}$ in the neighborhood of 0 , the conclusion of Thm. 3.1 holds for $\widehat{X}$.

To prove this, the only new difficulty compared with the previous case is to check that the probability that the process jumps before being killed or hitting zero is smaller than $C x$ when $\widehat{X}_{0}=x$. By Condition $(\mathrm{C})$, this is equivalent to prove the same property for the process without killing. Since the first jump time is exponential of parameter 1 , this can be proved with the exact same argument as in Prop. 3.5 since $\tau_{\partial}^{c}=\int_{0}^{\infty} L_{T_{0}^{B}}^{y} d m(y)$.

Of course, many easy extensions are possible, for example with jumps from $x$ to $g(x)$ at constant rate, where $g$ is a non-decreasing function and might be 0 on some interval. In fact, even random jumps can be easily covered provided monotonicity properties of jump measures. This also covers situations with bounded non-decreasing jump rate. The case of general rates is more complicated but could also be attacked with our method.

4.2. Application to models of population dynamics. Quasi-stationary distributions for one-dimensional diffusion processes have attracted much interest as an application to population dynamics. In Cattiaux et al. (2009); Cattiaux and Méléard (2010), the authors consider the conditional behavior of diffusion processes conditioned not to hit 0. In Hening and Kolb (2014), the authors consider onedimensional diffusion processes that cannot hit 0 continuously ( 0 is a natural boundary) but are subject to a killing rate modeling the risk of catastrophic events. In this section, our aim is to bring together both points of view by considering logistic Feller diffusion processes and their extensions to general drifts, as in Cattiaux et al. (2009), with an addition killing rate.

More precisely, let us consider a diffusion process $Y$ on $[0,+\infty)$ solution to the SDE

$$
d Y_{t}=\sqrt{Y_{t}} d B_{t}+Y_{t} h\left(Y_{t}\right) d t
$$

where $h$ is a measurable function from $\mathbb{R}_{+} \rightarrow \mathbb{R}$ modeling the density dependence of the individual growth rate of the population. We assume that $Y$ is subject to the nonnegative killing rate $\kappa=\frac{d k_{Y}}{d m_{Y}} \in L_{\text {loc }}^{1}((0,+\infty))$. For such models, the conditions (D) and (D') directly give criteria for exponential convergence to the quasi-distribution. The next result gives explicit conditions on $\kappa$ for a large class of functions $h$. Other conditions on $\kappa$ could be obtained for other asymptotic behaviors of $h$.

Proposition 4.2. Assume that $h \in L_{l o c}^{1}([0,+\infty))$ and that there exists $C, \beta>0$ such that, for some $y_{0}>0$,

$$
h(y) \leq-C y^{\beta}, \quad \forall y \geq y_{0} .
$$

If $\kappa$ satisfies

$$
\int_{1}^{\infty} \frac{\kappa(y)}{y^{1+\beta}} d y<\infty
$$

and

$$
\text { either } \quad \int_{0}^{1} \frac{\kappa(y)}{y} d y<\infty \quad \text { or } \quad \limsup _{x \rightarrow 0} \kappa(x)<\infty
$$


then there exists a unique probability measure $\alpha$ on $(0,+\infty)$ and two constants $C, \gamma>0$ such that, for all initial distribution $\mu$ on $[0, \infty)$ with $\mu(\{0\})<1$,

$$
\left\|\mathbb{P}_{\mu}\left(X_{t} \in \cdot \mid t<\tau_{\partial}\right)-\alpha(\cdot)\right\|_{T V} \leq C e^{-\gamma t}, \forall t \geq 0 .
$$

Proof: In this case, the scale function and the speed measure associated with $Y$ are given by

$$
s(y)=\int_{0}^{y} e^{-2 \int_{0}^{u} h(z) d z} d u \quad \text { and } \quad m_{Y}(d y)=\frac{e^{2 \int_{0}^{y} h(u) d u}}{y} d y .
$$

The killing measure associated to $Y$ is $k_{Y}=\kappa(y) m(d y)=\frac{\kappa(y) e^{2 \int_{0}^{y} h(u) d u}}{y} d y$. Our aim is to prove that condition (D) or (D') holds true using Proposition 4.1.

To do so, let us first check that

$$
\int_{(0,+\infty)} s(y)\left(m_{Y}(d y)+k_{Y}(d y)\right)<\infty .
$$

We have

$$
\int_{(0,+\infty)} s(y)\left(m_{Y}(d y)+k_{Y}(d y)\right)=\int_{(0,+\infty)} \int_{0}^{y} e^{-2 \int_{0}^{u} h(z) d z} d u \frac{(1+\kappa(y)) e^{2 \int_{0}^{y} h(u) d u}}{y} d y .
$$

Since $h \in L_{\text {loc }}^{1}([0,+\infty))$, we have

$$
\int_{0}^{y} e^{-2 \int_{0}^{u} h(z) d z} d u \frac{(1+\kappa(y)) e^{2 \int_{0}^{y} h(u) d u}}{y} \sim_{y \rightarrow 0} 1+\kappa(y),
$$

which is integrable in any bounded neighborhood of 0 by assumption. As a consequence,

$$
\int_{\left(0, y_{0}\right)} s(y)\left(m_{Y}(d y)+k_{Y}(d y)\right)<\infty
$$

On the other hand,

$$
\begin{aligned}
e^{2 \int_{0}^{y} h(u) d u} \int_{0}^{y} e^{-2 \int_{0}^{u} h(z) d z} d u & =\int_{0}^{y} e^{2 \int_{u}^{y} h(z) d z} d u \\
& \leq \int_{0}^{y_{0}} e^{2 \int_{u}^{y} h(z) d z} d u+\int_{y_{0}}^{y} e^{-2 C /(1+\beta)\left(y^{1+\beta}-u^{1+\beta}\right)} d u .
\end{aligned}
$$

We have, for all $y \geq y_{0}$,

$$
\int_{0}^{y_{0}} e^{2 \int_{u}^{y} h(z) d z} d u \leq \int_{0}^{y_{0}} e^{2 \int_{u}^{y_{0}} h(z) d z} d u e^{-2 C /(1+\beta)\left(y^{1+\beta}-y_{0}^{1+\beta}\right)}=C^{\prime} e^{-2 C y^{1+\beta} /(1+\beta)}
$$

for some finite constant $C^{\prime}$. Moreover, setting $C_{\beta}=\sup _{x \in(0,1)}(1-x) /\left(1-x^{1+\beta}\right)<$ $\infty$, we have

$$
\begin{aligned}
\int_{y_{0}}^{y} e^{-2 C /(1+\beta)\left(y^{1+\beta}-z^{1+\beta}\right) d z} d u & \leq \int_{y_{0}}^{y} e^{-2 C /(1+\beta) C_{\beta}^{-1}(y-z) y^{\beta} d z} d u \\
& \leq \frac{(1+\beta) C_{\beta}}{2 C y^{\beta}} .
\end{aligned}
$$

As a consequence, there exists $C^{\prime \prime}>0$ such that

$$
\int_{\left(y_{0},+\infty\right)} s(y)\left(m_{Y}(d y)+k_{Y}(d y)\right) \leq C^{\prime \prime} \int_{\left(y_{0},+\infty\right)} \frac{1+\kappa(y)}{y^{1+\beta}} d y
$$

which is finite by assumption. Finally, we conclude that (4.3) holds. 
The diffusion process $X=s(Y)$ is on natural scale with speed measure $m_{X}=$ $m_{Y} * s$, which is dominated by $(2 / x) d \Lambda(x)$ in the neighborhood of 0 , since $s(y) \sim_{0+}$ $y$ and $s^{\prime}(y) \rightarrow_{0+}$. We deduce from Thm. 3.4 that $X$ satisfies (3.1) and hence that $Y$ satisfies

$$
\mathbb{P}\left(t_{1}<\widetilde{T}_{0} \mid Y_{0}=y\right) \leq A s(y), \quad \forall y>0 .
$$

On the one hand, if $\int_{0}^{1} \frac{\kappa(y)}{y} d y<\infty$ then $\int_{0}^{1} k_{Y}(d y)<\infty$, which allows us to conclude that (D) holds using Point (i) of Proposition 4.1. On the other hand, if $\limsup _{x \rightarrow 0} \kappa(x)<\infty$, then Point (iii) of the same proposition holds true and hence (D') holds.

4.3. On processes solutions to stochastic differential equations and comparison with the literature. In the case where the speed measure $m$ is absolutely continuous w.r.t. the Lebesgue measure on $(0, \infty)$, our diffusion processes on natural scale are solutions, before killing, to SDEs of the form

$$
d X_{t}=\sigma\left(X_{t}\right) d B_{t}
$$

where $\sigma$ is a measurable function from $(0, \infty)$ to itself such that the speed measure $m(d x)=\frac{1}{\sigma^{2}(x)} d x$ is locally finite on $(0, \infty)$. Following the scale function trick of Section 4.1, our results actually cover all SDEs of the form

$$
d Y_{t}=\sigma\left(Y_{t}\right) d B_{t}+b\left(Y_{t}\right) d t
$$

such that $b / \sigma^{2} \in L_{\text {loc }}^{1}((0, \infty))$ (see Chapter 23 of Kallenberg, 2002).

Existence of quasi-stationary distribution of diffusion processes with killing has been already studied in Steinsaltz and Evans (2007); Kolb and Steinsaltz (2012). These works are based on a careful study of the generator of the process and SturmLiouville methods. Their results cover the case of entrance or natural boundary at $\infty$, but only the case of regular boundary at 0 . Our results only cover the case of entrance boundary at $\infty$, but 0 can be either regular or exit. In the case where $\infty$ is an entrance boundary, the stronger previously known result is Kolb and Steinsaltz (2012, Thm. 4.13) for diffusion processes $\left(Y_{t}, t \geq 0\right)$ on $[0, \infty)$ which are solution to SDEs of the form

$$
d Y_{t}=d B_{t}-h\left(Y_{t}\right) d t
$$

with killing measure absolutely continuous w.r.t. the Lebesgue measure with continuous density on $[0, \infty)$. Let us recall that the scale function of the solution $\left(Y_{t}, t \geq 0\right)$ to $(4.5)$ is given by

$$
s(x)=\int_{0}^{x} \exp \left(2 \int_{1}^{y} h(z) d z\right) d y,
$$

and that $X_{t}=s\left(Y_{t}\right)$ is solution to the SDE

$$
d X_{t}=\exp \left(2 \int_{1}^{s^{-1}\left(X_{t}\right)} h(z) d z\right) d B_{t} .
$$

In particular, this diffusion with killing is not as general as (4.4) since it only covers the case where the diffusion coefficient is positive and continuous. In fact, in all the references previously cited, the drift $h$ is assumed at least continuous so that the diffusion coefficient in (4.6) is at least $C^{1}$. 
In addition, our criteria allow unbounded killing rates at 0 as shown in the next proposition, contrary to existing results. This result can be proved similarly as Proposition 4.2 .

Proposition 4.3. Assume that $h \in L_{l o c}^{1}([0,+\infty))$ and that there exists $C>0$ and $\beta>1$ such that, for some $y_{0}>0$,

$$
h(y) \leq-C y^{\beta}, \quad \forall y \geq y_{0} .
$$

If $\kappa$ satisfies

then (D) is satisfied.

$$
\int_{0}^{1} \kappa(y) d y<\infty \text { and } \int_{1}^{\infty} \frac{\kappa(y)}{y^{\beta}} d y<\infty
$$

In the case where $\kappa$ is continuous bounded and $h$ is continuous on $[0,+\infty)$, existence of a quasi-stationary distribution was already proved in Kolb and Steinsaltz (2012). However, even in this case, uniqueness of the QSD, exponential convergence of conditional distributions and exponential ergodicity of the $Q$-process are new. Conversely, other results of Kolb and Steinsaltz (2012) cannot be easily studied with our methods, such as dichotomy results on the behavior of conditioned diffusion processes with killing and the case of natural boundary at $\infty$.

\section{On continuous and discontinuous absorption times when 0 is regular or exit and $\infty$ is entrance}

We give in this section a result on the probability $\mathbb{P}_{x}\left(\tau_{\partial}^{c}<t \wedge \tau_{\partial}^{d}\right)$ that the process hits 0 continuously before being killed, which is useful for the proof of our results of Subsection 3.1. In the introduction, we called this property coming down from infinity before killing in reference to the classical property of coming down from infinity for diffusion processes without killing (see Cattiaux et al., 2009; Champagnat and Villemonais, 2016b). Since this result also has an interest by itself, we give it in a separate section.

\section{Theorem 5.1.}

$$
\begin{aligned}
\exists t>0 \text { such that } \lim _{x \rightarrow+\infty} \mathbb{P}_{x}\left(\tau_{\partial}^{c}<t \wedge \tau_{\partial}^{d}\right)>0 \\
\qquad \int_{0}^{\infty} y(d k(y)+d m(y))<\infty .
\end{aligned}
$$

This result is obtained as a consequence of the next proposition.

Proposition 5.2. For all $k$ and $m$ and all $t>0$,

$$
\mathbb{P}_{x}\left(\tau_{\partial}^{c}<t \wedge \tau_{\partial}^{d}\right)=\mathbb{P}_{x}\left(\tau_{\partial}^{c}<t\right)=\mathbb{E}\left[\mathbb{1}_{\int_{0}^{\infty} Z_{s}^{x} m(d s)<t} \exp \left(-\int_{0}^{\infty} Z_{s}^{x} k(d s)\right)\right],
$$

where

$$
Z_{t}^{x}=2 \int_{0}^{t} \sqrt{Z_{s}^{x}} d W_{s}+2(t \wedge x), \quad \forall t \geq 0 .
$$

In particular, $\mathbb{P}_{x}\left(\tau_{\partial}^{c}<t \wedge \tau_{\partial}^{d}\right)$ is non-increasing w.r.t. $x>0$ and

$$
\lim _{x \rightarrow+\infty} \mathbb{P}_{x}\left(\tau_{\partial}^{c}<t \wedge \tau_{\partial}^{d}\right)=\mathbb{E}\left[\mathbb{1}_{\int_{0}^{\infty} Z_{s} m(d s)<t} \exp \left(-\int_{0}^{\infty} Z_{s} k(d s)\right)\right],
$$

where $\left(Z_{t}, t \geq 0\right)$ is a squared Bessel process of dimension 2 started from 0. 
Proof: We have

$$
\begin{aligned}
\mathbb{P}_{x}\left(\tau_{\partial}^{c}<t \wedge \tau_{\partial}^{d}\right) & =\mathbb{P}_{x}\left(\widetilde{T}_{0}<t \text { and } \kappa_{\widetilde{T}_{0}}<\mathcal{E}\right) \\
& =\mathbb{P}_{x}\left(\int_{0}^{\infty} L_{\widetilde{T}_{0}}^{y} m(d y)<t \text { and } \int_{0}^{\infty} L_{\sigma_{T_{0}}}^{y} k(d y)<\mathcal{E}\right) \\
& =\mathbb{P}_{x}\left(\int_{0}^{\infty} L_{T_{0}^{B}}^{y} m(d y)<t \text { and } \int_{0}^{\infty} L_{T_{0}^{B}}^{y} k(d y)<\mathcal{E}\right) \\
& =\mathbb{E}_{x}\left[\mathbb{1}_{\int_{0}^{\infty} L_{T_{0}^{B}}^{y} m(d y)<t} \exp \left(-\int_{0}^{\infty} L_{T_{0}^{B}}^{y} k(d y)\right)\right] .
\end{aligned}
$$

Hence (5.2) follows from Ray-Knight's theorem (cf. e.g. Rogers and Williams, 2000).

Since the process $Y_{t}^{x}=\sqrt{Z_{t}^{x}}$ is solution to

$$
d Y_{t}^{x}=d W_{t}+\frac{2 \mathbb{1}_{t \leq x}-1}{2 Y_{t}^{x}} d t
$$

standard comparison arguments show that the processes $\left(Y^{x}\right)_{x \geq 0}$ constructed with the same Brownian motion satisfy $Y_{t}^{x} \leq Y_{t}^{x^{\prime}}$ a.s. for all $t \geq 0$ and $x \leq x^{\prime}$, and $Y_{t}:=\lim _{x \rightarrow+\infty} Y_{t}^{x}$ is a Bessel process of dimension 2. Eq. (5.3) then follows from Lebesgue's theorem.

Proof of Theorem 5.1: In view of (5.3), the equivalence (5.1) follows from Pitman and Yor (1982, Prop. (2.2), Lemma (2.3)), which states that, for any square Bessel process $\left(R_{t}, t \geq 0\right)$ of positive dimension started from 0 and any positive Radon measure $\mu$ on $(0,+\infty)$,

$$
\mathbb{P}\left(\int_{0}^{\infty} R_{t} d \mu(t)<\infty\right)=1 \quad \Longleftrightarrow \quad \int_{0}^{\infty} t d \mu(t)<\infty
$$

and

$$
\mathbb{P}\left(\int_{0}^{\infty} R_{t} d \mu(t)=\infty\right)=1 \Longleftrightarrow \int_{0}^{\infty} t d \mu(t)=\infty .
$$

Hence both r.v. $\int_{0}^{\infty} L_{T_{0}^{B}}^{y} m(d y)$ and $\int_{0}^{\infty} L_{T_{0}^{B}}^{y} k(d y)$ are a.s. finite iff $\int_{0}^{\infty} y(d k(y)+$ $d m(y))<\infty$, which entails (5.1).

\section{Proof of the results of Section 3}

Theorems 3.1 and 3.3 and the main part of Proposition 3.2 directly follow from the results on general Markov processes of Champagnat and Villemonais (2016a). More precisely, the following condition (A) is equivalent to (3.4) (Champagnat and Villemonais (2016a, Thm.2.1)), and implies properties (3.5) and (3.6) of Proposition 3.2 (Champagnat and Villemonais (2016a, Prop. 2.3)) and the whole Theorem 3.3 (Champagnat and Villemonais, 2016a, Thm. 3.1).

Assumption (A). There exists a probability measure $\nu$ on $(0,+\infty)$ such that

(A1) there exists $t_{0}, c_{1}>0$ such that for all $x>0$,

$$
\mathbb{P}_{x}\left(X_{t_{0}} \in \cdot \mid t_{0}<\tau_{\partial}\right) \geq c_{1} \nu(\cdot) ;
$$

(A2) there exists $c_{2}>0$ such that for all $x>0$ and $t \geq 0$,

$$
\mathbb{P}_{\nu}\left(t<\tau_{\partial}\right) \geq c_{2} \mathbb{P}_{x}\left(t<\tau_{\partial}\right) .
$$


Hence, we need first to prove that (C) implies (A) (in Subsection 6.1), second, to prove that (C') implies (A) (in Subsection 6.2), and finally, to prove that $\eta(x) \leq C x$ for all $x \geq 0$ (in Subsection 6.3), which is the only part of Proposition 3.2 left to prove.

6.1. Proof that Condition $(C)$ implies $(A)$. Note that Champagnat and Villemonais (2016a, Thm. 2.1) also assumes that

$$
\mathbb{P}_{x}\left(t<\tau_{\partial}\right)>0, \quad \forall x>0, \quad \forall t>0,
$$

which is entailed by Prop. 2.2.

Our proof of (A) follows four steps, similarly as the proof of Champagnat and Villemonais (2016b, Thm. 4.1). In the first step, we prove that when $X_{0}$ is close to 0 , then, conditionally on non-absorption, the process exits some neighborhood of 0 with positive probability in bounded time. In the second step, we construct the measure $\nu$ involved in (A). We next prove (A1) in the third step, and (A2) in the last step.

Step 1: the conditioned process escapes a neighborhood of 0 in finite time.

The goal of this step is to prove that there exists $\varepsilon, c>0$ such that

$$
\mathbb{P}_{x}\left(X_{t_{1}} \geq \varepsilon \mid t_{1}<\tau_{\partial}\right) \geq c, \quad \forall x>0,
$$

where $t_{1}$ is taken from Assumption (C).

To prove this, recall that $\widetilde{X}$ is a diffusion process on natural scale with speed measure $m$ but with null killing measure. We first observe that, since $\widetilde{X}$ is a local martingale, for all $x \in(0,1)$,

$$
x=\mathbb{E}_{x}\left(\widetilde{X}_{t_{1} \wedge \widetilde{T}_{1} \wedge \tau_{\kappa}}\right),
$$

where we recall that $\tau_{\kappa}=\tau_{\partial}^{d}$ on the event $\tau_{\partial}^{d}<\infty$ and $\widetilde{T}_{0}=\tau_{\partial}^{c}$ on the event $\tau_{\partial}^{c}<\infty$. But the absorption at 0 ensures that

$$
\widetilde{X}_{t_{1} \wedge \widetilde{T}_{1} \wedge \tau_{\kappa}}=\widetilde{X}_{t_{1} \wedge \widetilde{T}_{1} \wedge \tau_{\kappa}} \mathbb{1}_{t_{1} \wedge \tau_{\kappa}<\widetilde{T}_{0}}+\mathbb{1}_{\widetilde{T}_{1}<\widetilde{T}_{0}<t_{1} \wedge \tau_{\kappa}} .
$$

We thus have for all $x \in(0,1)$

$$
x=\mathbb{P}_{x}\left(t_{1} \wedge \tau_{\kappa}<\widetilde{T}_{0}\right) \mathbb{E}_{x}\left(\widetilde{X}_{t_{1} \wedge \widetilde{T}_{1} \wedge \tau_{\kappa}} \mid t_{1} \wedge \tau_{\kappa}<\widetilde{T}_{0}\right)+\mathbb{P}_{x}\left(\widetilde{T}_{1}<\widetilde{T}_{0}<t_{1} \wedge \tau_{\kappa}\right) .
$$

Now, the strong Markov property entails

$$
\mathbb{P}_{x}\left(\widetilde{T}_{1}<\widetilde{T}_{0}<t_{1} \wedge \tau_{\kappa}\right) \leq \mathbb{P}_{x}\left(\widetilde{T}_{1}<\widetilde{T}_{0}\right) \mathbb{P}_{1}\left(\widetilde{T}_{0}<t_{1} \wedge \tau_{\kappa}\right) .
$$

Since $\mathbb{P}_{1}\left(\widetilde{T}_{0}<t_{1} \wedge \tau_{\kappa}\right)<1$ (see Prop. 5.2) and $\mathbb{P}_{x}\left(\widetilde{T}_{1} \leq \widetilde{T}_{0}\right)=x$ (recall that $\tilde{X}$ is a local martingale), we deduce from the two previous equations that there exists a constant $A^{\prime}>0$ such that

$$
A^{\prime} x \leq \mathbb{P}_{x}\left(t_{1} \wedge \tau_{\kappa}<\widetilde{T}_{0}\right) \mathbb{E}_{x}\left(\widetilde{X}_{t_{1} \wedge \widetilde{T}_{1} \wedge \tau_{\kappa}} \mid t_{1} \wedge \tau_{\kappa}<\widetilde{T}_{0}\right) .
$$

By Assumption $(\mathrm{C})$, we have $\mathbb{P}_{x}\left(t_{1}<\widetilde{T}_{0}\right) \leq A x$ and $\mathbb{P}_{x}\left(\tau_{\partial}^{d}<\tau_{\partial}^{c}\right) \leq A x$. But, by definition of $\tau_{\partial}^{c}$ and $\tau_{\partial}^{d}$, we have $\left\{\tau_{\partial}^{d}<\tau_{\partial}^{c}\right\}=\left\{\tau_{\kappa}<\widetilde{T}_{0}\right\}$, so that

$$
\mathbb{P}_{x}\left(t_{1} \wedge \tau_{\kappa}<\widetilde{T}_{0}\right) \leq 2 A x
$$

As a consequence,

$$
\mathbb{E}_{x}\left(1-\widetilde{X}_{t_{1} \wedge \widetilde{T}_{1} \wedge \tau_{\kappa}} \mid t_{1} \wedge \tau_{\kappa}<\widetilde{T}_{0}\right) \leq 1-\frac{A^{\prime}}{2 A} .
$$


Since we can assume without loss of generality that $\frac{A^{\prime}}{2 A}<1$, Markov's inequality implies that, setting $b=1-\sqrt{1-A^{\prime} / 2 A}$,

$$
\mathbb{P}_{x}\left(\widetilde{X}_{t_{1} \wedge \widetilde{T}_{1} \wedge \tau_{\kappa}} \leq b \mid t_{1} \wedge \tau_{\kappa}<\widetilde{T}_{0}\right) \leq \frac{1-A^{\prime} / 2 A}{1-b}=1-b,
$$

hence

$$
\mathbb{P}_{x}\left(\widetilde{X}_{t_{1} \wedge \widetilde{T}_{1} \wedge \tau_{\kappa}}>b \mid t_{1} \wedge \tau_{\kappa}<\widetilde{T}_{0}\right) \geq b .
$$

We deduce that, for all $x \in(0, b)$,

$$
\mathbb{P}_{x}\left(\widetilde{T}_{b}<t_{1} \wedge \tau_{\kappa} \mid t_{1} \wedge \tau_{\kappa}<\widetilde{T}_{0}\right)=\mathbb{P}_{x}\left(\widetilde{T}_{b}<t_{1} \wedge \widetilde{T}_{1} \wedge \tau_{\kappa} \mid t_{1} \wedge \tau_{\kappa}<\widetilde{T}_{0}\right) \geq b .
$$

Now, since $\mathbb{P}_{b}\left(t_{1}<\widetilde{T}_{0} \wedge \tau_{\kappa}\right)>0$ (see Prop. 2.2), there exists $\varepsilon \in(0, b)$ such that

$$
\mathbb{P}_{b}\left(t_{1}<\widetilde{T}_{\varepsilon} \wedge \tau_{\kappa}\right)>0 .
$$

Hence, we deduce from the strong Markov property that

$$
\begin{aligned}
\mathbb{P}_{x}\left(X_{t_{1}} \geq \varepsilon\right) & =\mathbb{P}_{x}\left(\widetilde{X}_{t_{1}} \geq \varepsilon \text { and } t_{1}<\tau_{\kappa}\right) \\
& \geq \mathbb{P}_{x}\left(\widetilde{T}_{b}<t_{1} \wedge \tau_{\kappa}\right) \mathbb{P}_{b}\left(t_{1}<\widetilde{T}_{\varepsilon} \wedge \tau_{\kappa}\right) \\
& \geq b \mathbb{P}_{x}\left(t_{1} \wedge \tau_{\kappa}<\widetilde{T}_{0}\right) \mathbb{P}_{b}\left(t_{1}<\widetilde{T}_{\varepsilon} \wedge \tau_{\kappa}\right),
\end{aligned}
$$

where the last inequality follows from (6.3). But $\mathbb{P}_{x}\left(t_{1} \wedge \tau_{\kappa}<\widetilde{T}_{0}\right) \geq \mathbb{P}_{x}\left(t_{1}<\right.$ $\left.\widetilde{T}_{0} \wedge \tau_{\kappa}\right)=\mathbb{P}_{x}\left(t_{1}<\tau_{\partial}\right)$, so that

$$
\mathbb{P}_{x}\left(X_{t_{1}} \geq \varepsilon \mid t_{1}<\tau_{\partial}\right) \geq b \mathbb{P}_{b}\left(t_{1}<\widetilde{T}_{\varepsilon} \wedge \tau_{\kappa}\right)>0 .
$$

This entails (6.2) for $x<b$.

For $x \geq b$, the continuity (before killing) and the strong Markov property for $X$ imply

$$
\begin{aligned}
\mathbb{P}_{x}\left(X_{t_{1}}>\varepsilon \mid t_{1}<\tau_{\partial}\right) & \geq \mathbb{P}_{x}\left(X_{t_{1}}>\varepsilon\right) \\
& \geq \mathbb{P}_{x}\left(T_{b}<\infty\right) \mathbb{P}_{b}\left(t_{1}<\widetilde{T}_{\varepsilon} \wedge \tau_{\kappa}\right) \\
& \geq \mathbb{P}_{x}\left(\tau_{\partial}^{c}<\infty\right) \mathbb{P}_{b}\left(t_{1}<\widetilde{T}_{\varepsilon} \wedge \tau_{\kappa}\right)
\end{aligned}
$$

Using Proposition 5.2 and (6.4), the proof of (6.2) is completed.

Step 2: Construction of coupling measures for the unconditioned process.

We prove that there exist two constants $t_{2}, c^{\prime}>0$ such that, for all $x \geq \varepsilon$,

$$
\mathbb{P}_{x}\left(X_{t_{2}} \in \cdot\right) \geq c^{\prime} \nu
$$

where

$$
\nu=\mathbb{P}_{\varepsilon}\left(X_{t_{2}} \in \cdot \mid t_{2}<\tau_{\partial}\right) .
$$

Fix $x \geq \varepsilon$ and construct two independent diffusions $X^{\varepsilon}$ and $X^{x}$ with speed measure $m(d x)$, killing measure $k(d x)$, and initial value $\varepsilon$ and $x$ respectively. Let $\theta=\inf \left\{t \geq 0: X_{t}^{\varepsilon}=X_{t}^{x}\right\}$. By the strong Markov property, the process

$$
Y_{t}^{x}= \begin{cases}X_{t}^{x} & \text { if } t \leq \theta, \\ X_{t}^{\varepsilon} & \text { if } t>\theta\end{cases}
$$

has the same law as $X^{x}$. By the continuity of the paths of the diffusions before killing, $\theta \wedge \tau_{\partial}^{d, x} \leq \tau_{\partial}^{x}:=\inf \left\{t \geq 0: X_{t}^{x}=0\right\}$. Hence, for all $t>0$,

$$
\mathbb{P}(\theta<t) \geq \mathbb{P}\left(\tau_{\partial}^{c, x}<t \text { and } \tau_{\partial}^{c, x}<\tau_{\partial}^{d, x}\right) .
$$


By Theorem 5.1, there exists $t_{2}>0$ and $c^{\prime \prime}>0$ such that

$$
\inf _{y>0} \mathbb{P}_{y}\left(\tau_{\partial}^{c}<t_{2} \text { and } \tau_{\partial}^{c}<\tau_{\partial}^{d}\right) \geq c^{\prime \prime}>0
$$

Hence

$$
\begin{aligned}
\mathbb{P}_{x}\left(X_{t_{2}} \in \cdot\right) & =\mathbb{P}\left(Y_{t_{2}}^{x} \in \cdot\right) \geq \mathbb{P}\left(X_{t_{2}}^{\varepsilon} \in \cdot, \tau_{\partial}^{c, x}<t_{2} \text { and } \tau_{\partial}^{c, x}<\tau_{\partial}^{d, x}\right) \\
& \geq c^{\prime \prime} \mathbb{P}_{\varepsilon}\left(X_{t_{2}} \in \cdot\right)
\end{aligned}
$$

where the last inequality follows from the independence of $X^{x}$ and $X^{\varepsilon}$. Therefore, (6.5) is proved with $c^{\prime}=c^{\prime \prime} \mathbb{P}_{\varepsilon}\left(t_{2}<\tau_{\partial}\right)$.

Step 3: Proof of (A1).

Using successively the Markov property, Step 2 and Step 1, we have for all $x>0$

$$
\begin{aligned}
\mathbb{P}_{x}\left(X_{t_{1}+t_{2}} \in \cdot \mid t_{1}+t_{2}<\tau_{\partial}\right) & \geq \mathbb{P}_{x}\left(X_{t_{1}+t_{2}} \in \cdot \mid t_{1}<\tau_{\partial}\right) \\
& \geq \int_{\varepsilon}^{\infty} \mathbb{P}_{y}\left(X_{t_{2}} \in \cdot\right) \mathbb{P}_{x}\left(X_{t_{1}} \in d y \mid t_{1}<\tau_{\partial}\right) \\
& \geq c^{\prime} \nu(\cdot) \mathbb{P}_{x}\left(X_{t_{1}} \geq \varepsilon \mid t_{1}<\tau_{\partial}\right) \\
& \geq c c^{\prime} \nu(\cdot) .
\end{aligned}
$$

This entails (A1) with $t_{0}=t_{1}+t_{2}$ and $c_{1}=c c^{\prime}$.

Step 4: Proof of (A2).

Let $a>0$ be such that $\nu([a,+\infty))>0$. Then we have, for all $x \geq a$,

$$
\begin{aligned}
\mathbb{P}_{x}\left(X_{t_{0}} \geq a\right) & \geq c_{1} \nu([a,+\infty)) \mathbb{P}_{x}\left(\tau_{\partial}>t_{0}\right) \\
& \geq c_{1} \nu([a,+\infty)) \mathbb{P}_{x}\left(T_{a}<\infty\right) \mathbb{P}_{a}\left(\tau_{\partial}>t_{0}\right) \\
& \geq c_{1} \nu([a,+\infty)) \mathbb{P}_{x}\left(\tau_{\partial}^{c}<\tau_{\partial}^{d}\right) \mathbb{P}_{a}\left(\tau_{\partial}>t_{0}\right),
\end{aligned}
$$

where we have used the strong Markov property for the second inequality. By Theorem 5.1, $\inf _{x \in(0,+\infty)} \mathbb{P}_{x}\left(\tau_{\partial}^{c}<\tau_{\partial}^{d}\right)>0$ and we know that $\mathbb{P}_{a}\left(\tau_{\partial}>t_{0}\right)>0$ by Proposition 2.2. As a consequence,

$$
\inf _{x \geq a} \mathbb{P}_{x}\left(X_{t_{0}} \geq a\right)>0 .
$$

Using this inequality and a standard renewal argument, we deduce that there exists $\rho>0$ such that, for all $k \in \mathbb{N}$,

$$
\mathbb{P}_{a}\left(X_{k t_{0}} \geq a\right) \geq e^{-\rho k t_{0}} .
$$

Now, we know from Step 4 of the proof of Champagnat and Villemonais (2016b, Theorem 4.1) (see also Cattiaux et al., 2009) that, since the diffusion $\widetilde{X}$ on $[0, \infty$ ) without killing has $\infty$ as an entrance boundary, for all $r>0$, there exists $y_{r}>0$ (which can be assumed larger than $a$ without loss of generality) such that

$$
\sup _{x \geq y_{r}} \mathbb{E}_{x}\left(e^{r \widetilde{T}_{y_{r}}}\right)<+\infty .
$$

This implies

$$
\sup _{x \geq y_{\rho}} \mathbb{E}_{x}\left(e^{\rho\left(T_{y_{\rho}} \wedge \tau_{\partial}\right)}\right)<+\infty
$$


for the constant $\rho$ of (6.6). Using the strong Markov property, for all $x \in[a,+\infty)$ and $y \in\left[a, y_{\rho}\right]$,

$$
\begin{aligned}
\mathbb{P}_{x}\left(t<\tau_{\partial}\right) & \geq \mathbb{P}_{x}\left(T_{a}<\infty\right) \mathbb{P}_{a}\left(T_{y}<\infty\right) \mathbb{P}_{y}\left(t<\tau_{\partial}\right) \\
& \geq \mathbb{P}_{x}\left(\tau_{\partial}^{c}<\tau_{\partial}^{d}\right) \mathbb{P}_{a}\left(T_{y_{\rho}}<\infty\right) \mathbb{P}_{y}\left(t<\tau_{\partial}\right) .
\end{aligned}
$$

Using Theorem 5.1 and Proposition 2.2, we infer that there exists $C>0$ such that, for all $t \geq 0$,

$$
\sup _{x \in\left[a, y_{\rho}\right]} \mathbb{P}_{x}\left(t<\tau_{\partial}\right) \leq C \inf _{x \in[a,+\infty)} \mathbb{P}_{x}\left(t<\tau_{\partial}\right) .
$$

Finally, we also deduce from the Markov property that, for all $s<t$,

$$
\mathbb{P}_{a}\left(X_{\left\lceil s / t_{0}\right\rceil t_{0}} \geq a\right) \inf _{x \in[a,+\infty)} \mathbb{P}_{x}\left(t-s<\tau_{\partial}\right) \leq \mathbb{P}_{a}\left(t<\tau_{\partial}\right) .
$$

Now, for all $x \geq y_{\rho}$, with a constant $C>0$ that may change from line to line, using successively (6.7), (6.8), (6.9) and (6.6), we obtain

$$
\begin{aligned}
\mathbb{P}_{x}\left(t<\tau_{\partial}\right) & \leq \mathbb{P}_{x}\left(t<T_{y_{\rho}} \wedge \tau_{\partial}\right)+\int_{0}^{t} \mathbb{P}_{y_{\rho}}\left(t-s<\tau_{\partial}\right) \mathbb{P}_{x}\left(T_{y_{\rho}} \in d s\right) \\
& \leq C e^{-\rho t}+C \int_{0}^{t} \mathbb{P}_{a}\left(t-s<\tau_{\partial}\right) \mathbb{P}_{x}\left(T_{y_{\rho}} \in d s\right) \\
& \leq C e^{-\rho\left\lceil t / t_{0}\right\rceil t_{0}}+C \mathbb{P}_{a}\left(t<\tau_{\partial}\right) \int_{0}^{t} \frac{1}{\mathbb{P}_{a}\left(X_{\left\lceil s / t_{0}\right\rceil t_{0}} \geq a\right)} \mathbb{P}_{x}\left(T_{y_{\rho}} \in d s\right) \\
& \leq C \mathbb{P}_{a}\left(t<\tau_{\partial}\right)+C \mathbb{P}_{a}\left(t<\tau_{\partial}\right) \int_{0}^{t} e^{\rho s} \mathbb{P}_{x}\left(T_{y_{\rho}} \in d s\right) .
\end{aligned}
$$

We deduce that, for all $t \geq 0$,

$$
\sup _{x \in\left[y_{\rho},+\infty\right)} \mathbb{P}_{x}\left(t<\tau_{\partial}\right) \leq C \inf _{x \in[a,+\infty)} \mathbb{P}_{x}\left(t<\tau_{\partial}\right) .
$$

Now, for all $x \in[a,+\infty)$ and all $y \in(0, a)$,

$$
\begin{aligned}
\mathbb{P}_{x}\left(t<\tau_{\partial}\right) & \geq \mathbb{P}_{x}\left(T_{y}<\infty\right) \mathbb{P}_{y}\left(t<\tau_{\partial}\right) \\
& \geq \mathbb{P}_{x}\left(\tau_{\partial}^{c}<\tau_{\partial}^{d}\right) \mathbb{P}_{y}\left(t<\tau_{\partial}\right) .
\end{aligned}
$$

Using Proposition 5.2, we deduce that there exists $C>0$ such that, $\forall t \geq 0$,

$$
\sup _{x \in(0, a)} \mathbb{P}_{x}\left(t<\tau_{\partial}\right) \leq C \inf _{x \in[a,+\infty)} \mathbb{P}_{x}\left(t<\tau_{\partial}\right) .
$$

Finally, since $\nu([a,+\infty))>0,(6.8),(6.10)$ and (6.11) entail (A2).

6.2. Proof that Condition ( $\left.C^{\prime}\right)$ implies (A). The proof follows exactly the same steps as in Subsection 6.1. The only step which needs to be modified is Step 1, so we only detail this step.

Step 1: the conditioned process escapes a neighborhood of 0 in finite time.

The goal of this step is to prove that there exists $\varepsilon^{\prime}, c>0$ such that

$$
\mathbb{P}_{x}\left(X_{t_{1}} \geq \varepsilon^{\prime} \mid t_{1}<\tau_{\partial}\right) \geq c, \quad \forall x>0 .
$$

We know from the study of diffusions without killing of Champagnat and Villemonais (2016b, Step 1 of Section 5.1) that there exists $c, b>0$ such that, for all $x \leq b$,

$$
\mathbb{P}_{x}\left(\widetilde{T}_{b}<t_{1} \wedge \widetilde{T}_{0}\right) \geq c \mathbb{P}_{x}\left(t_{1}<\widetilde{T}_{0}\right),
$$


By Assumption (C') and using (2.1), a.s. for all $t \leq \widetilde{T}_{\varepsilon}$,

$$
\kappa_{t}=\int_{0}^{\infty} L_{\sigma_{t}}^{y} d k(y) \leq A \int_{0}^{\infty} L_{\sigma_{t}}^{y} d m(y)=A A_{\sigma_{t}}=A t .
$$

Note that we can assume without loss of generality in (6.13) that $b \leq \varepsilon$. Therefore, for all $x \in(0, b)$, a.s. under $\mathbb{P}_{x}$,

$$
\left\{\tau_{\kappa}>t_{1} \wedge \widetilde{T}_{b}\right\}=\left\{\kappa_{t_{1} \wedge \widetilde{T}_{b}}<\mathcal{E}\right\} \supset\left\{t_{1} \wedge \widetilde{T}_{b}<\mathcal{E} / A\right\} \supset\left\{t_{1}<\mathcal{E} / A\right\} .
$$

Hence, using (6.13) and the independence between $\mathcal{E}$ and $\widetilde{X}$,

$$
\begin{aligned}
\mathbb{P}_{x}\left(T_{b}<t_{1} \wedge \tau_{\partial}\right) & =\mathbb{P}_{x}\left(\left\{\widetilde{T}_{b}<t_{1} \wedge \widetilde{T}_{0}\right\} \cap\left\{\tau_{\kappa}>t_{1} \wedge \widetilde{T}_{b}\right\}\right) \\
& \geq \mathbb{P}_{x}\left(\left\{\widetilde{T}_{b}<t_{1} \wedge \widetilde{T}_{0}\right\} \cap\left\{t_{1}<\mathcal{E} / A\right\}\right) \\
& \geq c \mathbb{P}_{x}\left(t_{1}<\widetilde{T}_{0}\right) e^{-A t_{1}} \geq c e^{-A t_{1}} \mathbb{P}_{x}\left(t_{1}<\tau_{\partial}\right) .
\end{aligned}
$$

But, for all $\varepsilon^{\prime} \in(0, b), x \in(0, b)$,

$$
\mathbb{P}_{x}\left(X_{t_{1}} \geq \varepsilon^{\prime}\right) \geq \mathbb{P}_{x}\left(T_{b}<t_{1} \wedge \tau_{\partial}\right) \mathbb{P}_{b}\left(t_{1}<T_{\varepsilon^{\prime}} \wedge \tau_{\kappa}\right) .
$$

By Proposition 2.2, the last factor of the r.h.s. is positive for $\varepsilon^{\prime}>0$ small enough, which concludes the proof of (6.12), since the case $x \geq b$ can be handled exactly as in Subsection 6.1.

6.3. Proof that $\eta(x) \leq C x$ for all $x \geq 0$. We recall that, since we already proved that (C) and (C') imply (A), Champagnat and Villemonais (2016a, Prop. 2.3) entails the main part of Proposition 3.2, and we only have to check that there exists a constant $C$ such that $\eta(x) \leq C x$ for all $x \geq 0$, where $\eta(x)$ is the uniform limit of $e^{\lambda_{0} t} \mathbb{P}_{x}\left(t<\tau_{\partial}\right)$. For all $t \geq t_{1}$, where the constant $t_{1}$ is the one given in Assumption $(\mathrm{C})$ or $\left(\mathrm{C}^{\prime}\right)$,

$$
e^{\lambda_{0} t} \mathbb{P}_{x}\left(t<\tau_{\partial}\right)=e^{\lambda_{0} t} \mathbb{E}_{x}\left[\mathbb{P}_{X_{t_{1}}}\left(t-t_{1}<\tau_{\partial}\right)\right] \leq C e^{\lambda_{0} t_{1}} \mathbb{P}_{x}\left(t_{1}<\tau_{\partial}\right) .
$$

For the last inequality, we used the fact that $e^{\lambda_{0} s} \mathbb{P}_{y}\left(s<\tau_{\partial}\right)$ is uniformly bounded in $y>0$ and $s \geq 0$ because of the uniform convergence in (3.5).

By Assumption $(\mathrm{C})$ or $\left(\mathrm{C}^{\prime}\right), \mathbb{P}_{x}\left(t_{1}<\tau_{\partial}\right) \leq \mathbb{P}_{x}\left(t_{1}<\widetilde{T}_{0}\right) \leq A x$, and we obtain the inequality $\eta(x) \leq C x$ by letting $t \rightarrow+\infty$ in (6.14).

\section{References}

P. Cattiaux, P. Collet, A. Lambert, S. Martínez, S. Méléard and J. San Martín. Quasi-stationary distributions and diffusion models in population dynamics. Ann. Probab. 37 (5), 1926-1969 (2009). MR2561437.

P. Cattiaux and S. Méléard. Competitive or weak cooperative stochastic LotkaVolterra systems conditioned on non-extinction. J. Math. Biol. 60 (6), 797-829 (2010). MR2606515.

N. Champagnat and D. Villemonais. Exponential convergence to quasi-stationary distribution and Q-process. Probab. Theory Related Fields 164 (1-2), 243-283 (2016a). MR3449390.

N. Champagnat and D. Villemonais. Uniform convergence of conditional distributions for absorbed one-dimensional diffusions. ArXiv Mathematics e-prints (2016b). arXiv: 1506.02385. 
P. Collet, S. Martínez and J. San Martín. Quasi-stationary distributions. Probability and its Applications (New York). Springer, Heidelberg (2013). ISBN 978-3-642-33130-5; 978-3-642-33131-2. MR2986807.

E. A. van Doorn and P. K. Pollett. Quasi-stationary distributions for discrete-state models. European J. Oper. Res. 230 (1), 1-14 (2013). MR3063313.

W. Feller. The parabolic differential equations and the associated semi-groups of transformations. Ann. of Math. (2) 55, 468-519 (1952). MR0047886.

D. Freedman. Brownian motion and diffusion. Springer-Verlag, New York-Berlin, second edition (1983). ISBN 0-387-90805-6. MR686607.

A. Hening and M. Kolb. A stochastic lotka-volterra model with killing. ArXiv Mathematics e-prints (2014). arXiv: 1409.2387.

K. Itô and H. P. McKean Jr. Diffusion processes and their sample paths. SpringerVerlag, Berlin-New York (1974). MR0345224.

O. Kallenberg. Foundations of modern probability. Probability and its Applications (New York). Springer-Verlag, New York, second edition (2002). ISBN 0-38795313-2. MR1876169.

M. Kolb and D. Steinsaltz. Quasilimiting behavior for one-dimensional diffusions with killing. Ann. Probab. 40 (1), 162-212 (2012). MR2917771.

J. Littin. Uniqueness of quasistationary distributions and discrete spectra when $\infty$ is an entrance boundary and 0 is singular. J. Appl. Probab. 49 (3), 719-730 (2012). MR3012095.

S. Méléard. Application du calcul stochastique à l'etude de processus de Markov réguliers sur [0,1]. Stochastics 19 (1-2), 41-82 (1986). MR864337.

S. Méléard and D. Villemonais. Quasi-stationary distributions and population processes. Probab. Surv. 9, 340-410 (2012). MR2994898.

Y. Miura. Ultracontractivity for Markov semigroups and quasi-stationary distributions. Stoch. Anal. Appl. 32 (4), 591-601 (2014). MR3219695.

J. Pitman and M. Yor. A decomposition of Bessel bridges. Z. Wahrsch. Verw. Gebiete 59 (4), 425-457 (1982). MR656509.

P. K. Pollett. Quasi-stationary distributions: A bibliography (2015). Available on-line at http://www.maths.uq.edu.au/pkp/papers/qsds/.

D. Revuz and M. Yor. Continuous martingales and Brownian motion, volume 293 of Grundlehren der Mathematischen Wissenschaften [Fundamental Principles of Mathematical Sciences]. Springer-Verlag, Berlin, third edition (1999). ISBN 3540-64325-7. MR1725357.

L. C. G. Rogers and D. Williams. Diffusions, Markov processes, and martingales. Vol. 1. Cambridge Mathematical Library. Cambridge University Press, Cambridge (2000). ISBN 0-521-77594-9. MR1796539.

D. Steinsaltz and S. N. Evans. Quasistationary distributions for one-dimensional diffusions with killing. Trans. Amer. Math. Soc. 359 (3), 1285-1324 (2007). MR2262851. 